\title{
Palmitic Acid Downregulates Thyroglobulin (Tg), Sodium Iodide Symporter (NIS), and Thyroperoxidase (TPO) in Human Primary Thyrocytes: A Potential Mechanism by Which Lipotoxicity Affects Thyroid?
}

\author{
Meng Zhao $\mathbb{D}^{1,2,3}$ Xiaohan Zhang, ${ }^{1,2,3}$ Ling Gao $\mathbb{D}^{1,2,3,4}$ Yongfeng Song $\mathbb{D}^{1,2,3}$ Chao Xu, ${ }^{1,2,3}$ \\ Chunxiao $Y u,{ }^{1,2,3}$ Shanshan Shao $\mathbb{D}^{1,2,3}$ and Jiajun Zhao $\mathbb{D}^{1,2,3}$ \\ ${ }^{1}$ Department of Endocrinology, Shandong Provincial Hospital Affiliated to Shandong University, Jinan, Shandong 250021, China \\ ${ }^{2}$ Shandong Provincial Key Laboratory of Endocrinology and Lipid Metabolism, Jinan, Shandong 250021, China \\ ${ }^{3}$ Institute of Endocrinology and Metabolism, Shandong Academy of Clinical Medicine, Jinan, Shandong 250021, China \\ ${ }^{4}$ Scientific Center, Shandong Provincial Hospital Affiliated to Shandong University, Jinan, Shandong 250021, China
}

Correspondence should be addressed to Shanshan Shao; shaoshanshan11@126.com and Jiajun Zhao; jjzhao@sdu.edu.cn

Received 17 May 2018; Accepted 31 July 2018; Published 17 October 2018

Academic Editor: Claudio Casella

Copyright (c) 2018 Meng Zhao et al. This is an open access article distributed under the Creative Commons Attribution License, which permits unrestricted use, distribution, and reproduction in any medium, provided the original work is properly cited.

\begin{abstract}
Our previous studies suggested that the thyroid might be a target organ affected by lipotoxicity, which might be partially related to the increasing prevalence of subclinical hypothyroidism. However, the underlying molecular mechanism is not yet clearly established. This study aimed to assess the effect of palmitic acid stimulation on thyrocyte function. Upon palmitic acid stimulation, intracellular contents of lipids, as well as the expression and activity of three key molecules in thyroid hormone synthesis (i.e., thyroglobulin, sodium iodide symporter, and thyroperoxidase), were determined in human primary thyrocytes. The contents of BODIPY ${ }^{\circledR}$ FL C16 (the fluorescently labeled palmitic acid analogue) entering into the thyrocytes were gradually increased with time extending. Accordingly, the intracellular accumulation of both triglyceride and free fatty acids increased in dose- and time-dependent manners. The effect of palmitic acid stimulation on thyroid hormone synthesis was then determined. Both the mRNA and protein levels of thyroglobulin, sodium iodide symporter, and thyroperoxidase were decreased following palmitic acid stimulation. Consistently, upon palmitic acid stimulation, the secreted thyroglobulin levels in supernatants, ${ }^{131} \mathrm{I}$ uptake, and extracellular thyroperoxidase activity were all decreased in a dose-dependent manner. Our results demonstrated that upon palmitic acid stimulation, the expressions of the key molecules (thyroglobulin, sodium iodide symporter, and thyroperoxidase) were reduced and their activities were suppressed, which might lead to impaired thyroid hormone synthesis.
\end{abstract}

\section{Introduction}

Hypothyroidism, which is defined as failure of thyroid gland to produce sufficient thyroid hormone to meet the metabolic demands of the body, does affect a considerable proportion of the population [1-4]. A survey carried out in 10 cities in China indicated that the prevalence of hypothyroidism had dramatically increased from $4.33 \%$ in 1999 to $17.73 \%$ in 2011 [5]. It is universally accepted that primary hypothyroidism can result from congenital abnormalities, autoimmune destruction (Hashimoto disease), iodine deficiency, iatrogenic injury, and infiltrative diseases, among which autoimmune thyroid disease is the most common etiology of primary hypothyroidism [6]. However, risk factors contributing to the increasing prevalence of hypothyroidism have not been fully confirmed.

Lipotoxicity has attracted great attention worldwide for its serious and extensive impact on human health. In recent years, lipotoxicity has been well documented in the pathogenesis of type 2 diabetes mellitus, metabolic syndrome, and nonalcoholic fatty liver disease $[7,8]$. Furthermore, in the early 1990s, lipotoxicity induced by dietary fat overload 
TABle 1: Primers for quantitative real-time PCR in the study.

\begin{tabular}{lcccc}
\hline & NM & Product $(\mathrm{bp})$ & Forward $\left(5^{\prime}\right.$ to $\left.3^{\prime}\right)$ & Reverse $\left(5^{\prime}\right.$ to $\left.3^{\prime}\right)$ \\
\hline Human NIS & 000453.2 & 180 & GTCCTTCAGGGCTCCTTCACC & CTGCTCGCTGGGTGGGTACA \\
Human TPO & 000547.5 & 241 & CTGTCTGTCACGCTGGTTATGG & TCACTCCGCTTGTTGGCTCA \\
Human Tg & 003235.4 & 203 & TTCTTTGAATGTGAACGACGGTG & AAGGGATAGGTGTGGACTTCAATGT \\
Human BiP & 005347.4 & 150 & GCCTGTATTTCTAGACCTGCC & TTCATCTTGCCAGCCAGTTG \\
Human $\beta$-actin & 001101 & 104 & ACAGAGCCTCGCCTTTGCCG & ACATGCCGGAGCCGTTGTCG \\
\hline
\end{tabular}

NIS: sodium iodide symporter; TPO: thyroperoxidase; Tg: thyroglobulin; BiP: binding immunoglobulin protein.

was reported to interfere with the endocrine system [9]. Our previous studies suggested that the thyroid might be another target organ affected by lipotoxicity, which might be partially related to the increasing prevalence of subclinical hypothyroidism $[10,11]$. However, the underlying molecular mechanism is not yet clearly established.

Here, we explored the effect of palmitic acid (PA) stimulation on thyrocyte. This study provides a more comprehensive understanding of the pathophysiological effects of lipotoxicity on thyroid function.

\section{Materials and Methods}

2.1. Human Primary Thyrocyte Culture. Human thyroid samples were obtained from patients undergoing partial thyroidectomy for treatment of benign follicular nodules at Shandong Provincial Hospital affiliated to Shandong University from December 2016 to May 2017. Both men and women were adopted and each individual underwent serum thyroid function and antibody testing and Doppler ultrasonography. Subjects with thyroid tumor, thyroid dysfunction, and autoimmune thyroid disease were all excluded. The Ethics Committee of Shandong Provincial Hospital approved the experimental protocol, and all participants provided written informed consent before collecting samples. According to the standardized procedure [12], all of the obtained specimens were dissected from the region most distant from the affected tissue and were digested in $2 \mathrm{mg} / \mathrm{ml} \mathrm{col-}$ lagenase I (Sigma-Aldrich) and $0.125 \%$ trypsin for 1 hour. After filtering and washing, thyrocytes were seeded on culture dishes (Corning). The medium consisted of DMEM/F12 medium (HyClone) supplemented with $10 \%$ newborn calf serum (GIBCO), bovine TSH $(2 \mathrm{mU} / \mathrm{ml}$; Sigma-Aldrich), and penicillin-streptomycin $(100 \mathrm{U} / \mathrm{ml})$. The cells were cultured in a humidified incubator containing 95\% air and 5\% $\mathrm{CO}_{2}$ at $37^{\circ} \mathrm{C}$ until cell attachment. The culture medium was replaced with fresh medium every 24 hours. As appropriate for the experiments to be performed, the cells were washed twice with PBS and then starved in serum-free medium overnight before they were treated with palmitic acid (Sigma-Aldrich).

2.2. Intracellular Localization of BODIPY® FL $C_{16}$. Human primary thyrocytes were washed with PBS and incubated with $1 \mu \mathrm{M}$ BODIPY ${ }^{\circledR}$ FL C $_{16}$ (Life Technologies), the fluorescently labeled palmitic acid analogue in $0.1 \%$ DMSO, pH 7.2 at $37^{\circ} \mathrm{C}$ for 5 minutes, 15 minutes, and 30 minutes, respectively. Then, the thyrocytes were briefly rinsed with PBS and fixed with $4 \%$ paraformaldehyde for $15 \mathrm{~min}$ at $37^{\circ} \mathrm{C}$. Nuclei were stained with 4, 6-diamidino-2-phenylindole (DAPI; Vector Laboratories) and the resultant immunofluorescence was viewed under a fluorescent microscope (Axio Imager 2, Carl Zeiss, Jena, Germany).

2.3. Determination of Intracellular Triglyceride and Free Fatty Acid. Human primary thyrocytes were incubated in DMEM/ F12 medium containing 10\% newborn calf serum until $80 \%$ confluency was achieved. The cells were subsequently changed to serum-free medium and treated without or with different concentrations of palmitic acid $(0.2$ or $0.4 \mathrm{mM})$ for the indicated time intervals ( 12 or 48 hours). The intracellular triglyceride and free fatty acid was extracted and measured using a Triglyceride Quantization Kit (Applygen Technologies) and a Free Fatty Acid Quantization Kit (Biovision) according to the manufacturer's instructions. All data were normalized with the protein concentration in a parallel well.

2.4. Quantitative Real-Time PCR. Quantitative real-time PCR was performed according to a previously described method [13]. Total RNA from cells was isolated using Trizol reagent (Takara, Tokyo, Japan), following the manufacturer's instructions. RT reaction $(20 \mu \mathrm{l})$ was carried out using $1 \mathrm{ug}$ of total RNA, oligo-dT primer, random 6-mers, and reverse transcriptase (RT) (Takara). Real-time PCR was performed in LC480 (Roche, Mannheim, Germany) according to instructions. SYBR green (Takara) was used to detect the amplification of cDNA in a total volume of $20 \mu \mathrm{l}$ with the absolute quantitative, $\Delta \mathrm{Ct}$ method. Each reaction consisted of $10 \mu \mathrm{l}$ of SYBR green, $1 \mu \mathrm{l}$ of cDNA sample, $2 \mu \mathrm{l}$ of each primer pair $(5 \mu \mathrm{M})$, and $7 \mu \mathrm{l}$ of distilled water. Thermal cycling conditions were $5 \mathrm{~min}$ at $95^{\circ} \mathrm{C}$ followed by 40 cycles at $95^{\circ} \mathrm{C}$ for $10 \mathrm{sec}, 60^{\circ} \mathrm{C}$ for $10 \mathrm{sec}$, and $72^{\circ} \mathrm{C}$ for $10 \mathrm{sec}$. The PCR primers were shown in Table $1 . \beta$-Actin was employed as an endogenous control to normalize the data. The specificity of the PCR amplification was verified by melting curve analysis of the final products (cooling the sample to $60^{\circ} \mathrm{C}$ and heating slowly to $95^{\circ} \mathrm{C}$ with measurement of fluorescence) and $10 \mu \mathrm{l}$ loaded on a $3 \%$ agarose gel to determine the size and specificity of the PCR product. All data were relative to control.

2.5. Western Blot Analysis. Equal amounts of protein from different samples were subjected to $10 \%$ SDS-PAGE, followed by electrotransfer from the gel to polyvinylidene difluoride membranes (Millipore). The membranes were incubated overnight at $4^{\circ} \mathrm{C}$ with anti-NIS $(1: 250$, Biorbyt $)$, 
anti-TPO ( $1: 200$, Santa Cruz), anti-Tg $(1: 10000$, Abcam), and anti-BiP (1:1000, Proteintech). GAPDH protein was evaluated as a loading control (1: 3500, Proteintech). Immune complexes were detected using the FluorChem Q Gel Imaging and Analysis System (Protein Simple, California, USA), and band densitometry was performed through the use of associated densitometry software (Protein Simple, California, USA).

2.6. Immunofluorescence. Human primary thyrocytes were treated without or with $0.2 \mathrm{mM}$ palmitic acid for 72 hours. The cells were washed with PBS and fixed with 4\% paraformaldehyde. Then, the cells were washed and blocked with $10 \%$ goat serum and incubated with anti-NIS (1:100, Biorbyt), anti-TPO (1:100, Santa Cruz), and anti-Tg (1:50, Abcam) at $4^{\circ} \mathrm{C}$ overnight, respectively. After further washing, the thyrocytes were incubated with secondary TRITC- or FITCconjugated goat anti-mouse IgG. Nuclei were stained with 4 , 6-diamidino-2-phenylindole (DAPI; Vector Laboratories) and the resultant immunofluorescence was viewed under a fluorescent microscope (Axio Imager 2, Carl Zeiss, Jena, Germany). All images were acquired using the same exposure time. As a negative control, the identical procedure was performed in the absence of the primary antibody and replaced by PBS. The semiquantitative analysis of fluorescence intensity was conducted using Image J software.

2.7. Detection of Secretory Thyroglobulin (Tg). Human primary thyrocytes were seeded in 6-well plates and treated without or with palmitic acid $(0.2$ or $0.4 \mathrm{mM})$ for 72 hours. The secretory $\mathrm{Tg}$ levels in supernatants were determined using chemiluminescent methods (Cobas E601; Roche, Basel, Switzerland) and normalized with corresponding protein amount.

2.8. Radioactive Iodide Uptake Assay. Human primary thyrocytes were seeded in 6-well plates and treated without or with palmitic acid ( 0.2 or $0.4 \mathrm{mM}$ ) for 72 hours. Radioactive iodide uptake assay was performed according to Zhang et al. previously described [14]. Briefly, cells were washed twice with Hanks' balanced salt solution and incubated with $2.0 \mu \mathrm{Ci}$ $\mathrm{Na}^{125} \mathrm{I}$ (Chengdu Gaotong Isotype Co. Ltd.) in $5 \mu \mathrm{M}$ nonradioactive $\mathrm{NaI}$ (Sigma-Aldrich) for 30 minutes at $37^{\circ} \mathrm{C}$ with $5 \% \mathrm{CO}_{2}$. The cells incubated in the presence of $80 \mu \mathrm{M}$ NIS inhibitor, perchlorate (Sigma-Aldrich), were determined simultaneously as blank controls. Cells were then washed twice with cold Hanks' balanced salt solution and lysed with $95 \%$ ethanol for $20 \mathrm{~min}$. The cell lysate was collected and radioactivity was counted by a $\gamma$-counter (MN-6110, Zonkia, Anhui, China). Radioactive iodide uptake values were normalized with corresponding cell counts. Experiments were performed in triplicate.

2.9. Enzymatic Activity of Extracellular TPO. Considering that TPO is expressed at the surface of the cell, we determined its extracellular enzymatic activity as previously described [15]. Human primary thyrocytes were seeded in 6 -well plates and treated without or with palmitic acid $(0.2$ or $0.4 \mathrm{mM})$ for 72 hours. The cells were washed twice with $2 \mathrm{ml}$ of PBS, then $0.5 \mathrm{ml}$ of reaction mixture was added

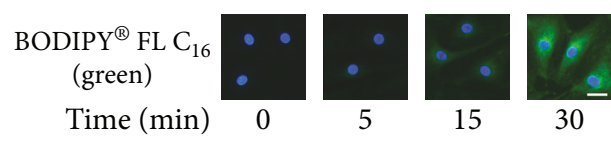

(a)
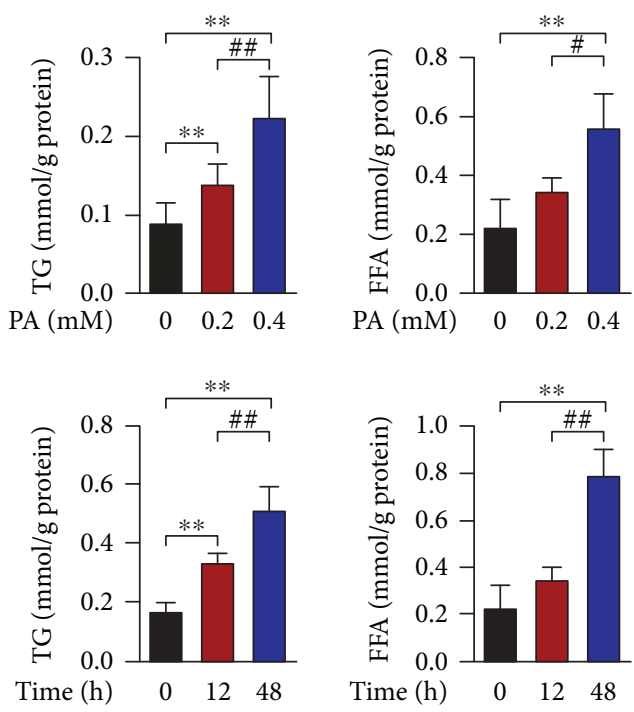

(b)

Figure 1: Palmitic acid increases the intracellular triglyceride and free fatty acid contents in human thyrocytes. (a) Intracellular distribution of BODIPY ${ }^{\circledR} \mathrm{FL} \mathrm{C}_{16}$, the fluorescently labeled palmitic acid analogue $(1 \mu \mathrm{M})$ in cells with time extending. Scale bars, $20 \mu \mathrm{m}$. (b) Upper: dose-dependent intracellular accumulations of triglyceride and free fatty acid in cells exposed to palmitic acid for 24 hours $(n=4)$. Lower: time-dependent intracellular accumulations of triglyceride and free fatty acid in cells exposed to palmitic acid at the concentration of $0.2 \mathrm{mM}(n=3)$. All data are expressed as the mean \pm standard deviation. ${ }^{* *} P<0.01$ versus human primary thyrocytes without palmitic acid stimulation. ${ }^{\#} P<0.05$ and ${ }^{\#} P<0.01$ versus human primary thyrocytes with palmitic acid stimulation at $0.2 \mathrm{mM}$ or for 12 hours. The error bars represent the standard deviations.

( $100 \mu \mathrm{M}$ KI (Sigma-Aldrich), $200 \mathrm{U} / \mathrm{ml}$ superoxide dismutase (SOD, Sigma-Aldrich), and $50 \mu \mathrm{M}$ Amplex Red (Life Technologies) in sodium phosphate buffer). The reaction was initiated by adding $25 \mu \mathrm{l}$ of $1 \mathrm{mM} \mathrm{H}_{2} \mathrm{O}_{2}$ (Sigma-Aldrich). $20 \mu \mathrm{l}$ of aliquots were removed at 1-minute intervals during 8 minutes and immediately mixed with $80 \mu \mathrm{l}$ of inhibition mixture containing $500 \mathrm{U} / \mathrm{ml}$ catalase (Sigma-Aldrich) and $100 \mathrm{U} / \mathrm{ml}$ SOD in PBS. The fluorescence was measured in a microtiter reader (Mithras ${ }^{2}$ LB 943, Berthold, Bad Wildbad, Germany) using excitation at $530 \mathrm{~nm}$ and emission at $590 \mathrm{~nm}$. Enzymatic activity values were normalized with corresponding protein amount.

2.10. Cell Viability Assay. Cell viability was assessed by Cell Counting Kit-8 (Dojindo, Japan) assay [16, 17]. Cells were seeded at 10000 cells/well into 96-well plates with $90 \mu \mathrm{l}$ culture medium. The $10 \mu \mathrm{l}$ of CCK- 8 solution was added to the cells at specific time points and cells were incubated for $2 \mathrm{hr}$ at $37^{\circ} \mathrm{C}$. The absorbance was measured at $450 \mathrm{~nm}$. The reaction product was quantified according to the manufacturer's instructions. 


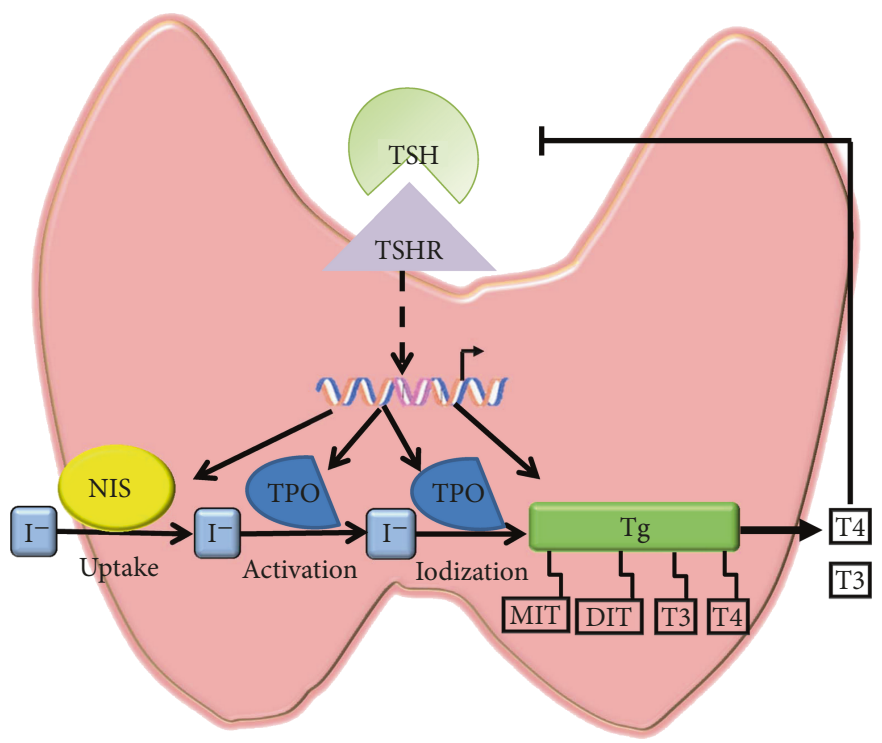

FIGURE 2: The process of thyroid hormone synthesis.

2.11. Statistical Analyses. Statistical tests were performed using SPSS version 18.0 for Windows (Chicago, IL, USA). Differences among control group and palmitic acid treatment groups were compared using one-way analysis of variance (ANOVA) (LSD or Dunnett's $t$-test) for multiple comparisons. The differences of extracellular TPO activity among control group and palmitic acid treatment groups were compared using repeated measurement data ANOVA. All of the calculated $P$ values were two sided, and $P$ values less than 0.05 were considered to be statistically significant.

\section{Results}

3.1. Excess Lipid Accumulation Induced by PA Stimulation in Human Primary Thyrocytes. PA, the most abundant saturated fatty acid found in diets and bloodstream, is well known to induce lipotoxic effect in various nonadipose cells and often employed to study the potential molecular mechanisms of cellular damage generated by lipotoxicity [18]. We determined whether PA was able to enter the human primary thyrocytes, which might be a prerequisite for lipotoxicity. As shown in Figure 1(a), the contents of BODIPY ${ }^{\circledR}$ FL $\mathrm{C}_{16}$ (the fluorescently labeled PA analogue) entering into the thyrocytes were gradually increased with time extending. Accordingly, the intracellular accumulation of both triglyceride and free fatty acids increased in dose- and timedependent manners (Figure 1(b)).

3.2. Expression Suppression of Key Molecules Involved in Thyroid Hormone Synthesis Induced by PA Stimulation in Human Primary Thyrocytes. Tg, NIS, and TPO are all key molecules in thyroid hormone synthesis (Figure 2) [19]. As shown in Figure 3(a), the mRNA levels of these three molecules decreased with the stimulation of PA. Moreover, their protein levels were also decreased significantly in human primary thyrocytes following PA stimulation (Figures 3(b) and 3(c) and Supplementary Figure 1), and the differences reached statistical significance (Figures 3(d) and 3(e)).

3.3. Activity Suppression of Key Molecules Involved in Thyroid Hormone Synthesis Induced by PA Stimulation in Human Primary Thyrocytes. As shown in Figure 2, Tg secretion, ${ }^{131}$ I uptake, and TPO catalysis are primary procedures in thyroid hormone synthesis. Consistently, in addition to the decreased expression, the activity of the three key molecules was also declined upon PA stimulation. As shown in Figure 4, the secreted Tg levels in supernatants and ${ }^{131} \mathrm{I}$ uptake were both decreased by approximately $30 \%$ in human primary thyrocytes stimulated with $0.4 \mathrm{mM}$ PA. Also, extracellular TPO activity was decreased in a dose-dependent manner.

3.4. Cell Viability Was Not Influenced by PA Stimulation in Human Primary Thyrocytes. To exclude the possibility that the reduction in expression and activity of key molecules was due to cell death, we performed cell viability assay. As shown in Supplementary Figure 2, cell viability was not influenced in human primary thyrocytes stimulated with $0.2 \mathrm{mM}$ PA $(P=0.361)$ or $0.4 \mathrm{mM} \mathrm{PA}(P=0.657)$ at any time point. Therefore, the reduced expression and activity of key molecules was not attributed to cytotoxicity.

3.5. Endoplasmic Reticulum Stress Was Induced by PA Treatment in Human Primary Thyrocytes. To explore the involved mechanism by which PA affects the three key molecules, we further observed the expression of binding immunoglobulin protein (BiP), a master regulator of unfolded protein response. As shown in Supplementary Figure 3, the mRNA and protein levels were both obviously increased upon PA stimulation in human primary thyrocytes. These results indicated that endoplasmic reticulum stress was induced by PA treatment in human primary thyrocytes. 

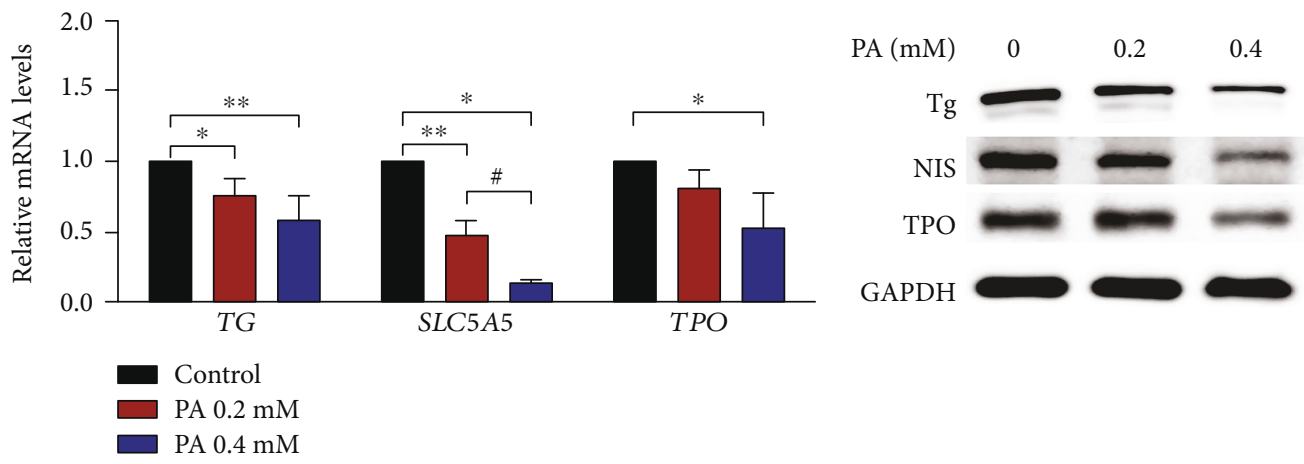

(a)
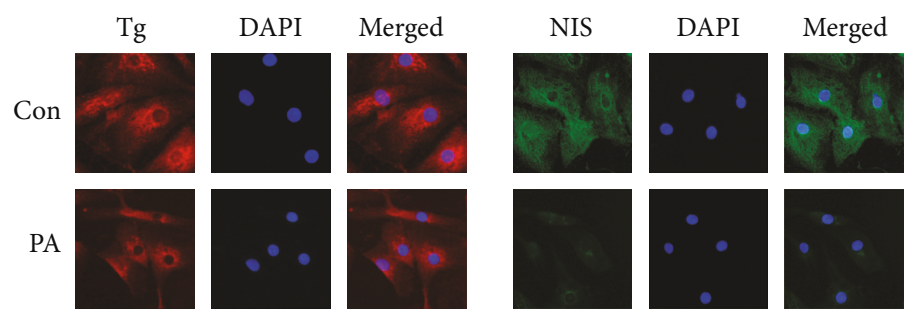

(b)

(c)
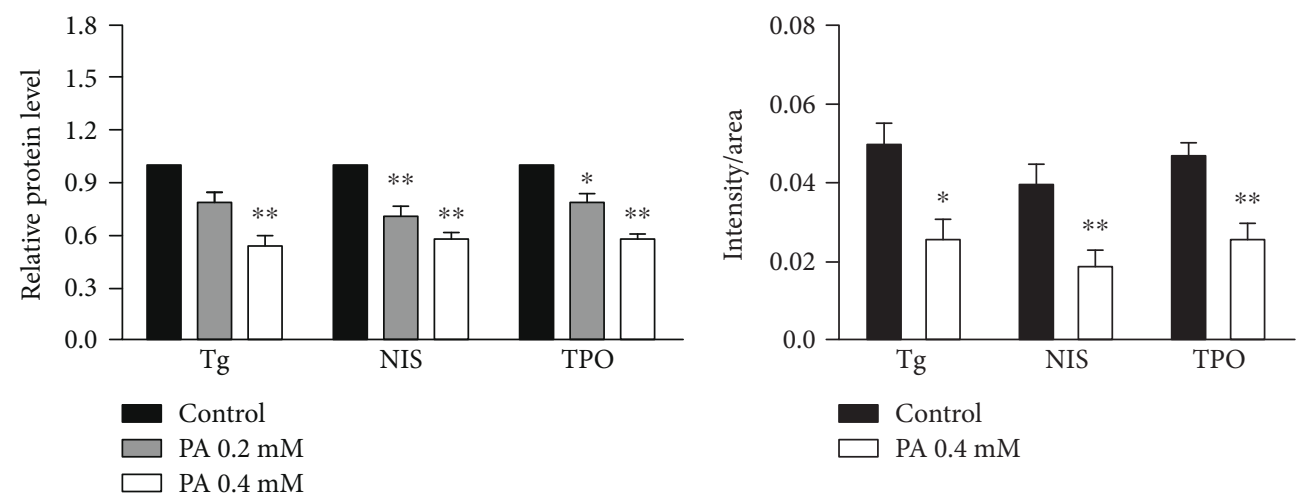

(d)

(e)

FIgure 3: Palmitic acid downregulates the expression of thyroglobulin (Tg), sodium iodide symporter (NIS), and thyroperoxidase (TPO) in human primary thyrocytes. (a) The mRNA levels of Tg, NIS (SLC5A5), and TPO in cells stimulated with palmitic acid for 24 hours ( $n=3$ to 5 ). (b) Dose-dependent effects of palmitic acid on the protein levels of Tg, NIS, and TPO for 72 hours detected by Western blot. (c) The protein levels of Tg, NIS, and TPO detected by immunofluorescence in cells stimulated with palmitic acid at $0.2 \mathrm{mM}$ for 72 hours. Scale bars, $20 \mu \mathrm{m}$. (d) The relative protein levels from Western blot assays for Tg, NIS, and TPO was quantified by densitometry and normalized with GAPDH. (e) The relative fluorescence intensity from immunofluorescence assays for Tg, NIS, and TPO. In all panels, representative data from 3 to 7 independent experiments are shown. All data are expressed as the mean \pm standard deviation. ${ }^{*} P<0.05$ and ${ }^{* *} P<0.01$ versus human primary thyrocytes without palmitic acid stimulation; ${ }^{\#} P<0.05$ versus human primary thyrocytes stimulated with palmitic acid at $0.2 \mathrm{mM}$. The error bars represent the standard deviations. PA: palmitic acid; Tg: thyroglobulin; SLC5A5: gene name of sodium iodide symporter; TPO: thyroperoxidase.

\section{Discussion}

Our results demonstrated that PA could induce excess intracellular lipid accumulation in human thyrocytes. Moreover, upon PA stimulation, the expressions of the key molecules were reduced and their activities were suppressed, which might lead to impaired thyroid hormone synthesis. Our study preliminarily suggests the involved mechanism by which lipotoxicity affects thyroid.

The main function of thyroid gland is to concentrate iodide and to make it available for biosynthesis of triiodothyronine (T3) and its precursor thyroxine (T4) [20]. In this process, a number of thyroid hormone synthesisrelated molecules were involved, such as $\mathrm{Tg}$, NIS, and TPO (Figure 2). NIS, locating in the basolateral membrane of follicular epithelial cells, transports iodate from bloodstream into the epithelial cells. Iodide enrichment mediated by NIS is the first step of thyroid hormone synthesis [21, 22]. TPO is located at the top of follicular epithelial cells. It catalyzes several crucial reactions, including activation of iodine, iodination of tyrosine residues, and coupling of iodinated tyrosine [19]. Furthermore, the main 


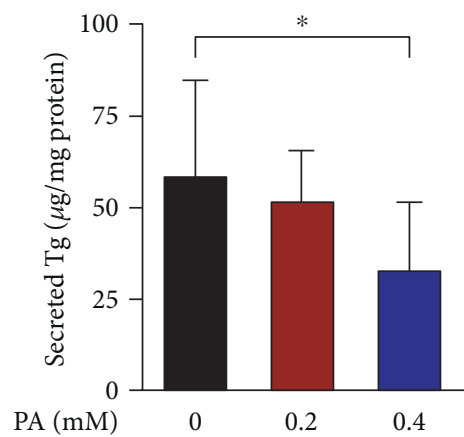

(a)

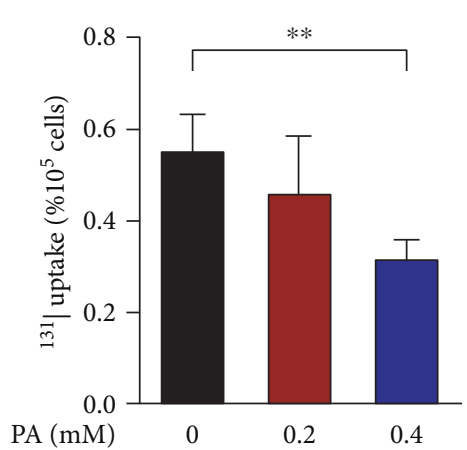

(b)

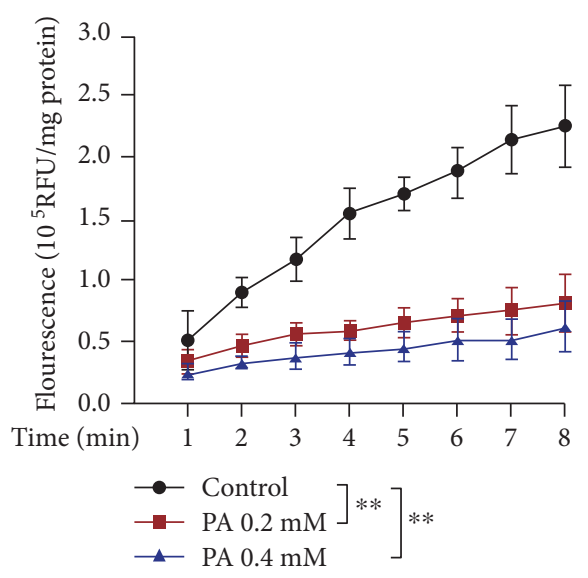

(c)

FIgure 4: Palmitic acid downregulates the activity of thyroglobulin (Tg), sodium iodide symporter (NIS), and thyroperoxidase (TPO) in human primary thyrocytes. (a) Secreted Tg levels in supernatants of cells stimulated with palmitic acid for 72 hours $(n=7)$. The Tg levels were normalized with corresponding protein amount. (b) The effects of palmitic acid stimulation on ${ }^{131} \mathrm{I}$ uptake for 72 hours $(n=7)$. ${ }^{131} \mathrm{I}$ uptake values were normalized with corresponding cell counts. (c) Extracellular TPO activity of the cells stimulated with palmitic acid for 72 hours $(n=6)$. Enzymatic activity values were normalized with corresponding protein amount. In all panels, representative data from 3 to 7 independent experiments are shown. All data are expressed as the mean \pm standard deviation. ${ }^{*} P<0.05$ and ${ }^{* *} P<0.01$ versus human primary thyrocytes without palmitic acid stimulation. The error bars represent the standard deviations. PA: palmitic acid; RFU: relative fluorescence unit.

function of iodinated tyrosine on $\mathrm{Tg}$ is to provide precursor substances for thyroid hormone synthesis, and it is also the storage form of thyroid hormone $[19,23]$. Since the above three molecules are involved in thyroid hormone synthesis, their suppressed expression and/or activity will decrease serum thyroid hormone concentration and finally lead to thyroid dysfunction.

Our previous studies demonstrated the effect of lipotoxicity on thyroid. In population, we found the positive association between hypertriglyceridemia and risk for subclinical hypothyroidism [10]. In rats, the high-fat lard diet decreased serum total T4 and free T4 levels in parallel with elevated serum thyroid-stimulating hormone levels [11]. However, the potential mechanism has not been fully elucidated. The present work suggested that lipotoxicity may induce hypothyroidism by suppressing the expression and/or activity of thyroid hormone synthesis, which is in agreement with some other studies. For instance, Xia et al. showed that NIS, TPO, and $\mathrm{Tg}$ mRNA levels were suppressed in obesity-prone mice fed a high-fat diet [24]. Interestingly, in Lee MH's research, although serum and intrathyroidal thyroxine levels were lower than those fed with normal chow diet, the expression of three key molecules was increased in diet-induced obese mice. This seemingly paradoxical elevation was speculated to result from the stimulatory actions of upregulated thyroid-stimulating hormone [25]. The above inconsistent observations suggest that further investigation is needed to clarify the complicated changes in thyroid with the stimulation of lipotoxicity.

Our study may provide novel clues for in-depth investigations to determine the possibly involved mechanism by which lipotoxicity affects thyroid. In Wen et al.'s study, endoplasmic reticulum stress was demonstrated to inhibit expression of Tg, NIS, and TPO in FRTL-5 thyrocytes [26]. Since
PA was also found to cause endoplasmic reticulum stress, we further investigated the change of $\mathrm{BiP}$ expression. Its upregulation indicated that endoplasmic reticulum stress was triggered in human primary thyrocytes stimulated by PA treatment. Similarly, in our animal experiments, endoplasmic reticulum stress was observed to be activated in male Sprague-Dawley rats fed with high-fat diet (data not shown). Thus, endoplasmic reticulum stress might mediate the effects of PA treatment on the suppression of three key molecules. In addition, sterol regulatory element-binding proteins (SREBPs), which were initially identified as master transcriptional regulators of lipid biosynthesis and uptake, were also reported to be novel transcriptional regulators of $\mathrm{Tg}$, NIS, and TPO in thyroid epithelial cells [27-29]. Other mechanism, such as mitochondrial oxidative stress or inflammation was also possible to be involved in the pathological process.

In summary, our study illustrated the suppressed expressions and activity of the key molecules (i.e., Tg, NIS, and TPO) in thyroid hormone synthesis upon PA stimulation. It is a preliminary exploration focusing on the mechanism by which lipotoxicity affects thyroid, and it provides novel clues for further investigations.

\section{Data Availability}

The data used to support the findings of this study are included within the supplementary information file(s).

\section{Ethical Approval}

The Ethics Committee of Shandong Provincial Hospital approved the experimental protocol, and all participants provided written informed consent before collecting samples. 


\section{Conflicts of Interest}

All authors (Meng Zhao, Xiaohan Zhang, Ling Gao, Yongfeng Song, Chao Xu, Chunxiao Yu, Shanshan Shao, and Jiajun Zhao) declared that they have no conflict of interest.

\section{Acknowledgments}

This research was supported by the National Natural Science Foundation (Grant nos. 81430020, 81670720, and 81300644) and the Research Fund for Public Welfare, National Health and Family Planning Commission of China (Grant no. 201402005).

\section{Supplementary Materials}

Figure S1: palmitic acid time-dependently downregulates the protein levels of thyroglobulin ( $\mathrm{Tg}$ ), sodium iodide symporter (NIS), and thyroperoxidase (TPO). Figure S2: cell viability was not influenced by palmitic acid stimulation in human primary thyrocytes. Figure S3: palmitic acid upregulates the expression of binding immunoglobulin protein $(\mathrm{BiP})$ in human primary thyrocytes. For all the readers to access the data supporting the conclusions of our study, we provided primary data of all the figures in the manuscript here. The means and standard deviations, shown in the figures, were both evaluated based on these data. (Supplementary Materials)

\section{References}

[1] W. M. G. Tunbridge, D. C. Evered, R. Hall et al., "The spectrum of thyroid disease in a community: the Whickham survey," Clinical Endocrinology, vol. 7, no. 6, pp. 481-493, 1977.

[2] C. T. Sawin, W. P. Castelli, J. M. Hershman, P. McNamara, and P. Bacharach, "The aging thyroid. Thyroid deficiency in the Framingham Study," Archives of Internal Medicine, vol. 145, no. 8, pp. 1386-1388, 1985.

[3] G. J. Canaris, N. R. Manowitz, G. Mayor, and E. C. Ridgway, "The Colorado thyroid disease prevalence study," Archives of Internal Medicine, vol. 160, no. 4, pp. 526-534, 2000.

[4] S. Zou, F. Wu, C. Guo et al., "Iodine nutrition and the prevalence of thyroid disease after salt iodization: a cross-sectional survey in Shanghai, a coastal area in China," PLoS One, vol. 7, no. 7, article e40718, 2012.

[5] Z. Shan, L. Chen, X. Lian et al., "Iodine status and prevalence of thyroid disorders after introduction of mandatory universal salt iodization for 16 years in China: a cross-sectional study in 10 cities," Thyroid, vol. 26, no. 8, pp. 1125-1130, 2016.

[6] P. A. Singer, "Thyroiditis. Acute, subacute, and chronic," The Medical Clinics of North America, vol. 75, no. 1, pp. 61-77, 1991.

[7] H. Shimano, "Novel qualitative aspects of tissue fatty acids related to metabolic regulation: lessons from Elovl6 knockout," Progress in Lipid Research, vol. 51, no. 3, pp. 267-271, 2012.

[8] J. D. McGarry, "Banting lecture 2001: dysregulation of fatty acid metabolism in the etiology of type 2 diabetes," Diabetes, vol. 51, no. 1, pp. 7-18, 2002.

[9] B. M. Tannenbaum, D. N. Brindley, G. S. Tannenbaum, M. F. Dallman, M. D. McArthur, and M. J. Meaney, "High-fat feeding alters both basal and stress-induced hypothalamicpituitary-adrenal activity in the rat," The American Journal of Physiology, vol. 273, 6 Part 1, pp. E1168-E1177, 1997.

[10] M. Zhao, X. Tang, T. Yang et al., "Lipotoxicity, a potential risk factor for the increasing prevalence of subclinical hypothyroidism?," The Journal of Clinical Endocrinology and Metabolism, vol. 100, no. 5, pp. 1887-1894, 2015.

[11] S. S. Shao, Y. F. Zhao, Y. F. Song et al., "Dietary high-fat lard intake induces thyroid dysfunction and abnormal morphology in rats," Acta Pharmacologica Sinica, vol. 35, no. 11, pp. 14111420, 2014.

[12] F. S. Ambesi-Impiombato, L. A. Parks, and H. G. Coon, "Culture of hormone-dependent functional epithelial cells from rat thyroids," Proceedings of the National Academy of Sciences of the United States of America, vol. 77, no. 6, pp. 3455-3459, 1980.

[13] Y. Sun, L. Zhang, H. F. Gu et al., "Peroxisome proliferatoractivated receptor-alpha regulates the expression of pancreatic/duodenal homeobox-1 in rat insulinoma (INS-1) cells and ameliorates glucose-induced insulin secretion impaired by palmitate," Endocrinology, vol. 149, no. 2, pp. 662-671, 2008.

[14] Z. Zhang, S. Beyer, and S. M. Jhiang, "MEK inhibition leads to lysosome-mediated $\mathrm{Na}^{+} / \mathrm{I}^{-}$symporter protein degradation in human breast cancer cells," Endocrine-Related Cancer, vol. 20, no. 2, pp. 241-250, 2013.

[15] M. Godlewska, M. Gora, A. M. Buckle et al., "A redundant role of human thyroid peroxidase propeptide for cellular, enzymatic, and immunological activity," Thyroid, vol. 24, no. 2, pp. 371-382, 2014.

[16] K. K. Huang, K. Ramnarayanan, F. Zhu et al., "Genomic and epigenomic profiling of high-risk intestinal metaplasia reveals molecular determinants of progression to gastric cancer," Cancer Cell, vol. 33, no. 1, pp. 137-150.e5, 2018.

[17] K. Chen, J. Liu, S. Liu et al., "Methyltransferase SETD2mediated methylation of STAT1 is critical for interferon antiviral activity," Cell, vol. 170, no. 3, pp. 492-506.e14, 2017.

[18] K. Ohtsubo, M. Z. Chen, J. M. Olefsky, and J. D. Marth, "Pathway to diabetes through attenuation of pancreatic beta cell glycosylation and glucose transport," Nature Medicine, vol. 17, no. 9, pp. 1067-1075, 2011.

[19] B. Czarnocka, "Thyroperoxidase, thyroglobulin, $\mathrm{Na}^{+} / \mathrm{I}^{-}$symporter, pendrin in thyroid autoimmunity," Frontiers in Bioscience, vol. 16, no. 1, pp. 783-802, 2011.

[20] C. G. P. Roberts and P. W. Ladenson, "Hypothyroidism," The Lancet, vol. 363, no. 9411, pp. 793-803, 2004.

[21] M. J. Willhauck, D. J. O`Kane, N. Wunderlich, B. Goke, and C. Spitzweg, "Stimulation of retinoic acid-induced functional sodium iodide symporter (NIS) expression and cytotoxicity of ${ }^{131}$ I by carbamazepine in breast cancer cells," Breast Cancer Research and Treatment, vol. 125, no. 2, pp. 377-386, 2011.

[22] A. Bizhanova and P. Kopp, "Minireview: the sodium-iodide symporter NIS and pendrin in iodide homeostasis of the thyroid," Endocrinology, vol. 150, no. 3, pp. 1084-1090, 2009.

[23] R. Fernando, S. Atkins, N. Raychaudhuri et al., "Human fibrocytes coexpress thyroglobulin and thyrotropin receptor," Proceedings of the National Academy of Sciences of the United States of America, vol. 109, no. 19, pp. 7427-7432, 2012.

[24] S. F. Xia, X. M. Duan, L. Y. Hao et al., "Role of thyroid hormone homeostasis in obesity-prone and obesity-resistant mice fed a high-fat diet," Metabolism, vol. 64, no. 5, pp. 566-579, 2015. 
[25] M. H. Lee, J. U. Lee, K. H. Joung et al., “Thyroid dysfunction associated with follicular cell steatosis in obese male mice and humans," Endocrinology, vol. 156, no. 3, pp. 1181-1193, 2015.

[26] G. Wen, R. Ringseis, and K. Eder, "Endoplasmic reticulum stress inhibits expression of genes involved in thyroid hormone synthesis and their key transcriptional regulators in FRTL-5 thyrocytes," PLoS One, vol. 12, no. 11, article e0187561, 2017.

[27] R. Ringseis, C. Rauer, S. Rothe et al., "Sterol regulatory element-binding proteins are regulators of the NIS gene in thyroid cells," Molecular Endocrinology, vol. 27, no. 5, pp. 781-800, 2013.

[28] C. Rauer, R. Ringseis, S. Rothe, G. Wen, and K. Eder, "Sterol regulatory element-binding proteins are regulators of the rat thyroid peroxidase gene in thyroid cells," PLoS One, vol. 9, no. 3, article e91265, 2014.

[29] G. Wen, K. Eder, and R. Ringseis, "Sterol regulatory elementbinding proteins are transcriptional regulators of the thyroglobulin gene in thyroid cells," Biochimica et Biophysica Acta, vol. 1859, no. 8, pp. 994-1003, 2016. 


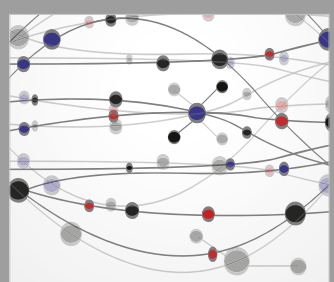

The Scientific World Journal
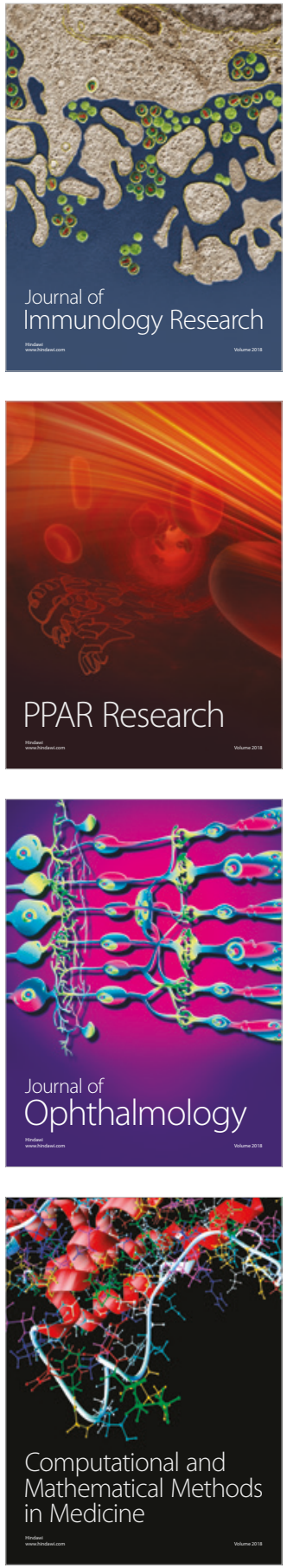

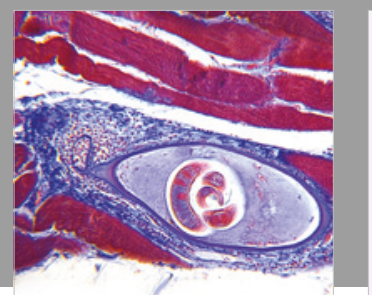

Gastroenterology Research and Practice

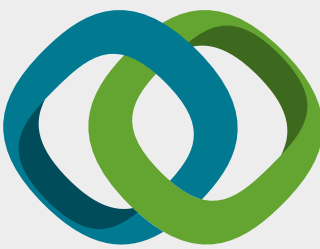

\section{Hindawi}

Submit your manuscripts at

www.hindawi.com
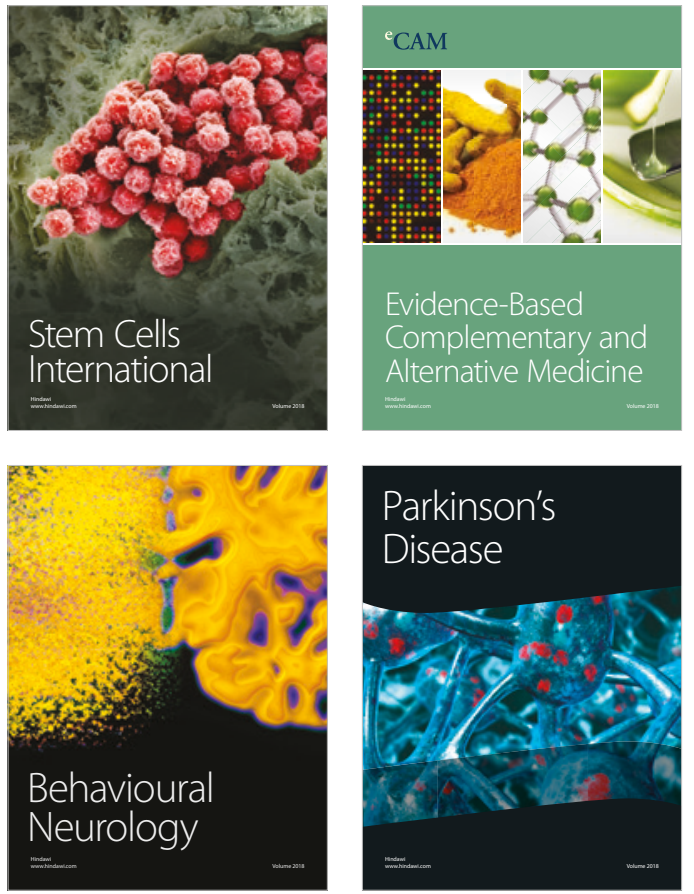

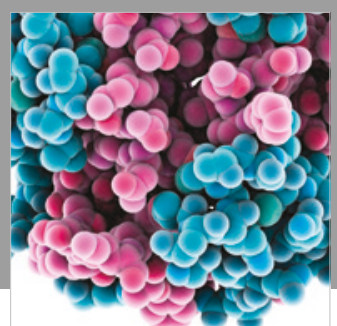

ournal of

Diabetes Research

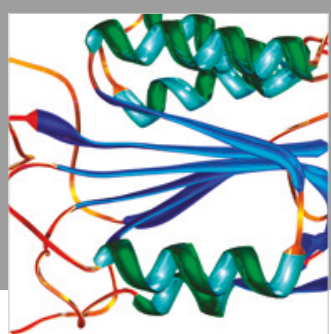

Disease Markers
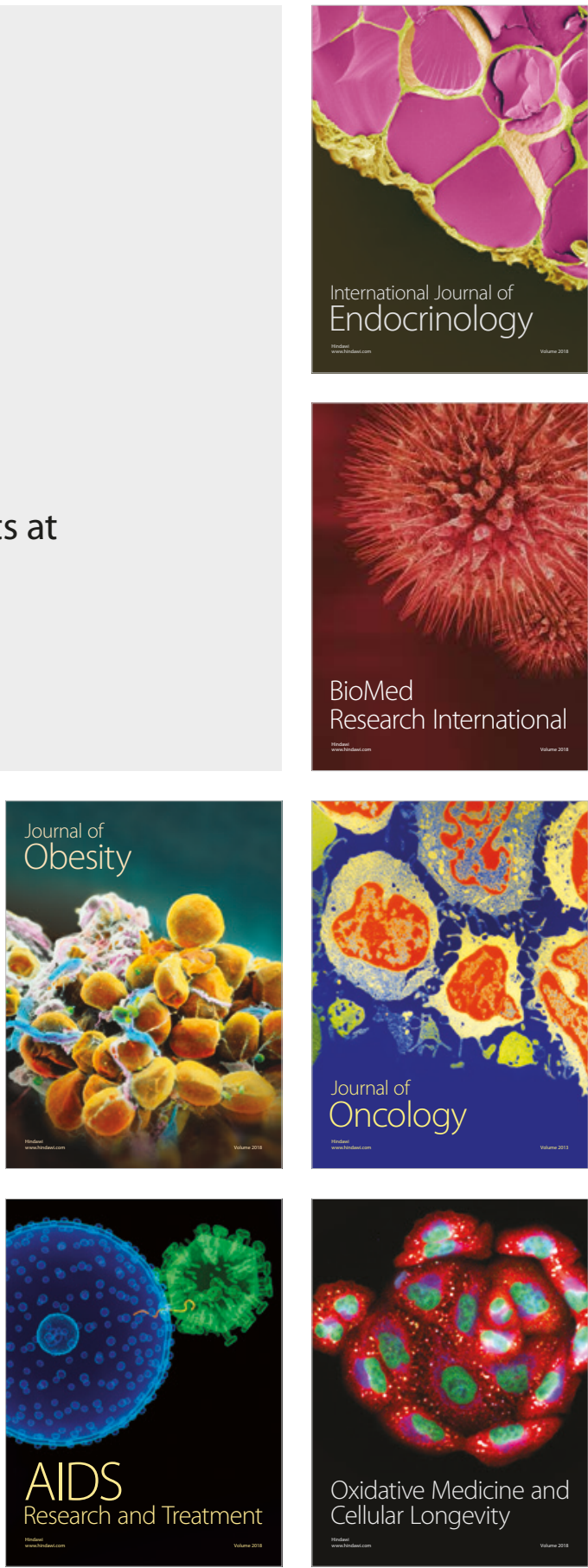